Nuclear Science and Technology Division

\title{
AUTONOMOUS CONTROL OF NUCLEAR POWER PLANTS
}

\author{
H. Basher* and J. S. Neal
}

October 2003

\author{
Prepared by \\ OAK RIDGE NATIONAL LABORATORY \\ Oak Ridge, Tennessee 37831-6283 \\ managed by \\ UT-Battelle, LLC \\ for the \\ U.S. DEPARTMENT OF ENERGY \\ under contract DE-AC-05-00OR22725
}

*Faculty Researcher from South Carolina State University. 



\section{CONTENTS}

\section{Page}

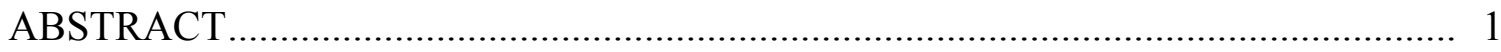

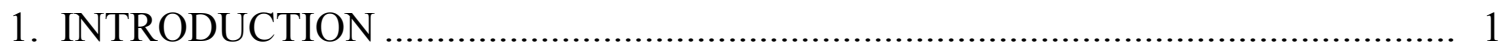

2. PRINCIPLES OF AUTONOMOUS CONTROL ................................................. 2

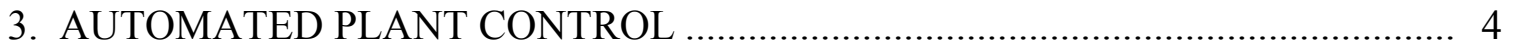

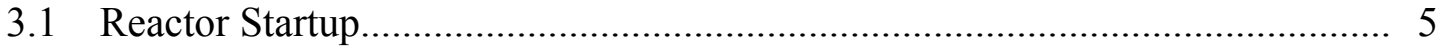

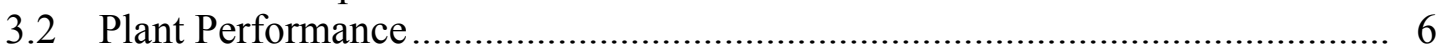

3.3 Fault-Tolerant Control ............................................................................. 7

3.4 Load-Following Operation.................................................................... 7

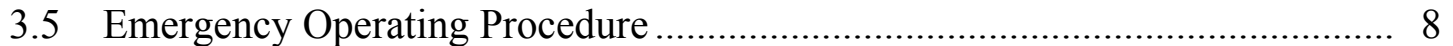

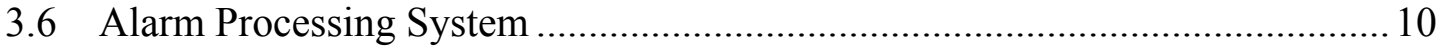

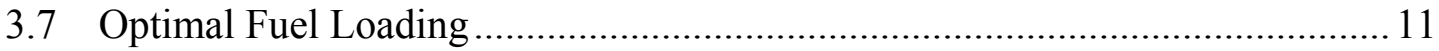

3.8 Fault Detection Identification .................................................................. 13

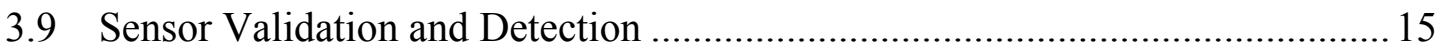

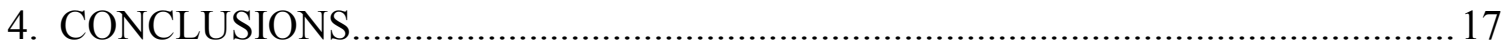

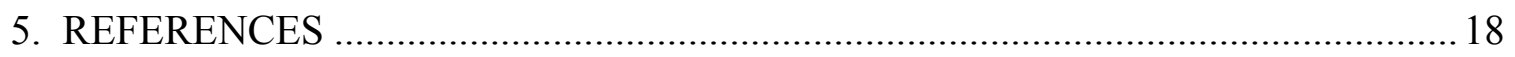





\title{
AUTONOMOUS CONTROL OF NUCLEAR POWER PLANTS H. Basher and J. S. Neal
}

\begin{abstract}
A nuclear reactor is a complex system that requires highly sophisticated controllers to ensure that desired performance and safety can be achieved and maintained during its operations. Higher-demanding operational requirements such as reliability, lower environmental impacts, and improved performance under adverse conditions in nuclear power plants, coupled with the complexity and uncertainty of the models, necessitate the use of an increased level of autonomy in the control methods. In the opinion of many researchers, the tasks involved during nuclear reactor design and operation (e.g., design optimization, transient diagnosis, and core reload optimization) involve important human cognition and decisions that may be more easily achieved with intelligent methods such as expert systems, fuzzy logic, neural networks, and genetic algorithms. Many experts in the field of control systems share the idea that a higher degree of autonomy in control of complex systems such as nuclear plants is more easily achievable through the integration of conventional control systems and the intelligent components. Researchers have investigated the feasibility of the integration of fuzzy logic, neural networks, genetic algorithms, and expert systems with the conventional control methods to achieve higher degrees of autonomy in different aspects of reactor operations such as reactor startup, shutdown in emergency situations, fault detection and diagnosis, nuclear reactor alarm processing and diagnosis, and reactor load-following operations, to name a few. With the advancement of new technologies and computing power, it is feasible to automate most of the nuclear reactor control and operation, which will result in increased safety and economical benefits. This study surveys current status, practices, and recent advances made towards developing autonomous control systems for nuclear reactors.
\end{abstract}

\section{INTRODUCTION}

Autonomous control systems have the potential for operating with very high levels of performance and reliability with a minimal or no human assistance. To be autonomous, a control system should provide adequate control actions in the presence of significant uncertainties. The key attributes of highly autonomous control systems are that they perform well under all process operating conditions and performance demands and are able to compensate for system failure without external intervention. More complex and sophisticated controllers are necessary to cope with changes in operating conditions relative to the reference model, the presence of large uncertainties in plant parameters and unmeasurable disturbances, the deterioration of components or component failures, etc. The conventional control techniques have evolved substantially over the past several decades from simple proportional-integral-derivative control to modern control such as optimal, adaptive, and robust control. With the development of optimal, adaptive, and robust control, 
the degree of autonomous operation of the controllers has increased. In order to further increase the availability and lower the accident risk and operating costs in nuclear power plants (NPPs), a higher level of automation and fault tolerance will be highly desirable.

Recent efforts have been directed toward the development of highly autonomous control systems that can independently perform complex tasks. This trend is gaining momentum as control engineers solve many existing problems and seek to resolve new control problems that involve broader issues and require the full capabilities of available computing technologies. Although it is clear that conventional control will play a major role in the development of such highly automated systems, the main focus in the control community is to integrate the functions of intelligent systems such as fuzzy logic, neural networks, genetic algorithms, and knowledge-based systems with the conventional control systems to perform complex tasks more easily. Intelligent control systems are typically able to perform one or more of the following functions to achieve autonomous behavior: planning actions at different levels of detail; emulating human expert behavior; learning from past experiences; integrating sensor information; identifying changes, such as failures, that threaten the system behavior; and reacting appropriately. The inclusion of the intelligent control aspect with conventional control techniques will encompass adaptation and learning; planning in the presence of large uncertainties; coping with huge data sets; and accommodation of plant dynamics, especially in complex nonlinear multivariable plants with numerous immeasurable parameters.

Uhrig $^{1}$ predicts the application of digital automation, including artificial intelligence techniques, to control and manage NPPs. He envisions that this automation will improve performance, increase safety margins, lower environmental impacts, and provide greater investment protection. This automation is attainable through further research, development, and advances in information processing technologies such as sensor validation, fault detection and diagnostics, fault-tolerant control, and intelligent systems. Some of the recent studies and/or experiments conducted to achieve higher autonomy in nuclear industries are highlighted in the following sections.

\section{PRINCIPLES OF AUTONOMOUS CONTROL}

An introduction to autonomous control may be found in refs. $2-13$. Antsaklis et al. ${ }^{2,3}$ have defined functions, characteristics, and behaviors of an autonomous system that could be used as guidelines in the design of such a system. They have also suggested that a hierarchical control structure is desirable to achieve an increasingly sophisticated autonomous controller. This architecture contains three levels: "execution level" (lowest), "coordination level," and "management and organization level" (highest). The general idea of the structure is that commands are issued by higher levels to lower levels and response data flows from lower levels to upper levels in the tier structure. The functions of each of these levels are described below. 
1. Execution level: The function of the execution level includes the generation of control actions as dictated by the higher levels and application of this control action to the actuators and sensors that drive the plant under control. The execution level also senses the plant responses and environment, processes them to identify parameters, detects any failures, and passes the information to the higher levels. This level possesses conventional adaptive control capabilities and includes failure detection algorithms, state estimators and parameter identifiers.

2. Coordination level: This level translates high-level commands into sequences of action to be passed to the execution level and provides the appropriate sequence of control and identification algorithms. The coordination level can cope with limited predetermined crisis situations.

3. Management and organization level: This level determines the system goals and supports the human communication interface, and oversees and directs all the activities at both the coordination and execution levels. It is the most intelligent of the three levels.

A similar three-tier structure is also proposed by Williams and Jouse. ${ }^{14}$ The proposed controller has been designed for the feedwater heater in an NPP.

It is an established fact that developments of optimal control, adaptive control, and robust control have significantly increased the level of automation by encompassing higher levels of plant characterization uncertainty and parameter changes. However, integration of intelligent components such as fuzzy logic, neural networks, and genetic algorithms, with the conventional control methodologies will further enhance the level of automation and permit this automation to be achieved more easily. ${ }^{11-13}$ On the other hand, some in the nuclear engineering community have raised concerns about this type of integration. ${ }^{8-10}$ Some suggest that conventional control has a better, more established track record than techniques from intelligent control, which are relatively new and in a very early stage of development. Stability analysis of a system being controlled is needed to verify the performance of the controller. Some progress has been made in the stability analysis of fuzzy logic, neural networks, and expert systems, but much more work is needed in the area of nonlinear analysis of intelligent control techniques. Validation of performance levels through experimental evaluations is needed. Above all, systematic controller design and construction methodologies are needed when intelligent control is used.

Ruan $^{15}$ summarizes the application of intelligent systems in nuclear reactors that was reported in the FLINS'96 (Fuzzy Logic and Intelligent Technologies in Nuclear Science) Workshop on Intelligent Systems and Soft Computing for Nuclear Science and Industry, held on September 25-27, 1996, in Mol, Belgium. The details of some of these presentations follow. In his paper, Nishiwaki proposed the use of fuzzy theory in application to failure analysis and diagnostics of NPPs, whereas Liu and Ruan discussed how the power control stability of the Belgian Reactor 1 (BR1) at the Research Center for the Applications of Nuclear Energy (SCK·CEN) was improved by using a fuzzy logic control scheme and described its potential to replace nuclear reactor operators in the control room. N.J. Na, et al. presented a real-time expert system for alarm processing and presentation in an NPP, 
which was tested in a simulated environment of the Three Mile Island (TMI)-2 accident. The inspection of anomalous symptoms and a diagnosis process on a simplified model of the steam generator feedwater systems of a pressurized heavy-water reactor (PHWR) using a knowledge-based system was presented by Guildo et al.

The activities reported in this paper deal with different aspects of nuclear operations, but they are all separate components of an autonomous controller for an NPP. For the sake of clarity, the discussions are grouped in areas such as automated plant control, automated startup, temperature performance, load-following operation, emergency operating procedure, alarm processing and diagnosis, optimal fuel loading, fault-tolerant control, fault detection and identification, and sensor signal validation. Since some investigations cover more than one aspect of NPP operations, a study included in a group may very well fit within another group as well.

\section{AUTOMATED PLANT CONTROL}

Man-machine interaction and dynamic process control of a pressurized-water nuclear reactor plant has been demonstrated by Cha. ${ }^{16}$ This process is developed by integrating real-time and knowledge-based agents and testing in a dynamic simulation environment. The goal is to use an experimental research test facility for process control, monitoring, and manmachine interface in the main control room of a nuclear reactor. The real time includes mathematical process models of the plant systems such as steam generator, feedwater system, control system, and electrical system. The knowledge-based agents are designed for process control, monitoring, procedural operator support, real-time diagnosis for control automatics, and user interface. The real-time knowledge-based system is evaluated under each of the simulated conditions such as the small-break loss-of-coolant accident (LOCA) and steam generator tube rupture (SGTR) and initial conditions such as startup, 10\% power and full power.

The studies conducted by Doraiswami et al. ${ }^{17}$ and Ben-Abdennour and Lee ${ }^{18}$ are not intended for NPP applications. However, these studies are still relevant because they propose and successfully implement systems in which the autonomy is enhanced through performance monitoring, fault diagnosing, and tuning the control system of the plants. Doraiswami provides a systematic and unified approach that accomplishes performance monitoring, performance improvement, and fault prediction in the control system. The controller is adapted to ensure acceptable performance for variations in the operating conditions. The signal estimation is performed in two steps. In the first step, a high-order model for the measurement record is obtained using the Linear Predictive Coding Algorithm. Then in the second step, a model reduction and validation is performed using an adaptive filtering algorithm. The controller tuning is performed using the steepest descent method. The proposed system is evaluated on an actual direct-drive robot manipulator made by Integrated Motions, Inc. The method is found to be extremely reliable in monitoring the plant performance, tuning the controller, and predicting the faults of physical systems. An autonomous controller developed for a boiler-turbine unit of a 160-MW oil-fired electric 
power plant is described in ref. 18. This approach realizes a fuzzy-logic-based intelligent coordinated control scheme. The control system performs two main tasks: execution and supervision. The execution is performed by the local controllers, and the supervision is carried out by a fuzzy-logic-based coordinator. The local controllers are of linear quadratic regulator/loop transfer recovery type, and an extended Kalman filter is used to observe the status of the nonlinear plant on-line. The residual error of the plant states is generated by comparing the estimates from the Kalman filter with the states of a nominal model. The residuals and any other possible disturbances are monitored by a fuzzy-logic-based coordinator. If a malfunction is detected, the coordinator modifies the set point of the local controllers to accommodate it.

An automated control engine that automatically provides a new control strategy under different operating conditions of a nuclear reactor is suggested by March-Leuba and Wood. ${ }^{19}$ The system is unique in the sense that it automatically optimizes the controller on-line for online plant parameter changes. This technique assumes that diagnostic methods that will identify component degradation or failure are developed and included in the system. In this study, the current operating conditions and status of failed components are passed on to the control engine by running the control engine and a diagnostic system in parallel with the real system. The plant model is updated on-line to incorporate the degradation or failure of components. The control engine then determines the new control parameters or chooses an alternate control algorithm such that plant performance requirements are satisfied with the new plant configuration. Prototype control engine software based on this concept is tested by running this with a high-fidelity pressurized-water reactor (PWR) simulator written in FORTRAN code. To avoid reactor scram, the technique is demonstrated for two transients characterized as a $10 \%$ power reduction and a $40^{\circ} \mathrm{F}$ reduction in feedwater temperature. The test results show that the system is very effective in maintaining the steam generator level and controlling the thermal power without oscillations.

\subsection{Reactor Startup}

If no disturbances occur, simply starting the appropriate systems in a fixed order is sufficient to start up the plant automatically. However, such a simple procedure cannot respond to the transients or abnormalities that may occur during startup. Some studies conducted to automate NPP startup operations are summarized in this section. Berkan et al. ${ }^{20}$ have proposed an automated startup control system for the Experimental Breeder Reactor-II (EBR-II), the performances of which are validated by comparing the operator-driven actual plant data with the simulation results. The simulations use a valid nonlinear model of the EBR-II. The range of the startup transients is chosen to be sufficiently broad to include nonlinear effects. The presence of plant nonlinearities over the startup of the reactor prompted the authors to use intelligent techniques such as reconstructive inverse dynamics, fuzzy logic, and neural networks to develop the controllers. The algorithms developed in this research validate sensor signals, strategies, commands, and performance tracking to emulate operator tasks, which, in turn, generate reliable decisions and control actions. The control module consists of three controllers designed on the basis of the above three techniques integrated to the existing conventional control. The performance of each of these controllers 
is satisfactory, providing fast and efficient diagnostics and reliable automatic control. A similar study of automated startup of the boiling water reactor (BWR) has been performed by Sekimizu et al. ${ }^{21}$ using knowledge-based techniques. The controller that contains an inference engine and an operation manager is tested with a BWR model (both written in LISP language). The results of the tests proved to be satisfactory.

\subsection{Plant Performance}

An automatically tuned fuzzy logic controller is proposed by Ramaswamy et al. ${ }^{22}$ to automate nuclear reactor operations for improved reactor temperature performance of a pressurized-water reactor (PWR) over the power range of $10-100 \%$ and with significant plant parameter variations, which will replace an existing optimal-state feedback controller. One of the unique features of this controller is that it uses a simple low-order observer to estimate the reactor temperature, compared with the full-state observer needed in the current controller. The existing adaptive controller requires a mathematical model of the reactor for its implementation, and its parameters are changed adaptively to cope with system nonlinearity and environmental variations. The fuzzy-logic-based controller, however, does not rely on an accurate description of the plant or the precise measurements, and its parameters are automatically tuned. Ramaswamy's paper gives detailed discussions of the various stages of the controller design process. The performance evaluation of the controller is conducted via simulations in which the simulated plant is a continuous-time point-kinetics model with six delayed neutron groups. The simulation results show that the autotuned fuzzy logic controller demonstrates good stability and robustness characteristics when compared with the adaptive controller for the same range of uncertainties such as power level variations of a factor of ten, and control rod worth variation of a factor of four.

The use of artificial neural networks (ANNs) can be found in many dynamic control systems, including nuclear reactors. Their results show that learning and interpolation abilities of neural networks are very promising. Several authors investigated the thermal power distribution in an NPP using a feedforward neural network (FNN), producing very encouraging results. FNN is a static mapping and requires a large number of neurons to represent a dynamic response in time domain, takes longer time to train, and does not guarantee convergence. The recurrent neural network (RNN) has improved performances compared with FNN but still is not very suitable because it is difficult to train and does not converge in a short time. On the other hand, diagonal recurrent neural retwork (DRNN) possesses a dynamic mapping capability, requires fewer neurons, and converges much faster. $\mathrm{Ku}$ et al..$^{23}$ have shown that by using DRNN instead of RNN, improved temperature response is attainable with higher reliability and lower computing cost. Their DRNN-based system contains a neurocontroller and a neuroidentifier, both of which are trained with a reference model that incorporates an optimal control law with improved reactor temperature response. The performances of the controller are demonstrated in a simplified model of a PWR, which is a point-kinetics model with one delayed neutron group and temperature feedback from a lumped fuel and coolant temperature calculation. The simulation results show that the controller performs very well not only locally but also over a wide range of operations. 
Fuzzy logic has been used in many NPP control problems. The main advantage of fuzzy logic in that once the membership functions and the rules are defined, the desired control signals can be easily generated using only elementary arithmetic operations. However, it is not always an easy job to find the membership functions and the rules, and usually a trialand-error method is used. To ease this process, some researchers have investigated the inclusion of neural networks to generate the membership functions and rules. One such controller that combines fuzzy logic and neural network methods to control core power distribution of NPPs was first reported in the work of Na and Upadhyaya. ${ }^{24}$ The neuro-fuzzy controller developed in this research does not require accurate description of the system but is based instead on expert knowledge of the plant. This controller automatically constructs and tunes the rule base and membership functions. The control method exhibits very fast response to any power offset without any residual oscillation. One unique feature of this controller is that the controller can be designed to be automatically fine-tuned to obtain the desired performance using the process data.

\subsection{Fault-Tolerant Control}

Stengel ${ }^{25}$ gives an overview of the characteristics of a fault-tolerant control system. He also explains why a fault-tolerant control is necessary and how such a system can be designed. Stengel's discussions emphasize again that the integration of conventional control and artificial intelligence components will dramatically enhance the system reconfiguration capability. This is further emphasized in the study performed by Rauch, ${ }^{26}$ who provides ideas on how fault diagnosis and control reconfiguration for complex systems can be achieved.

\subsection{Load-Following Operation}

Due to sharp variations in the power density during transients, the nuclear reactors exhibit a large local power peaking. In the manual core control system, the reactor operator chooses the best control rod maneuver procedure based on his knowledge and experience during this load-following operation. Research efforts have been focused on developing automatic control systems to improve this situation. The advances in conventional control, intelligent techniques, and speed of computing methods have enabled engineers to pursue a more sophisticated control system for load-following operation of nuclear reactors. Two of many works are cited here. ${ }^{27-28}$ The controller proposed by Khajavi et al. ${ }^{27}$ is based on neural networks and is trained from the response of a robust optimal self-tuning regulator. It is claimed that the controller has demonstrated a good stability and performance for a wide range of operation of a PWR whose model is of fifth order with one delayed neutron group and two thermal feedback mechanisms. One the other hand, a controller based on heuristic control algorithm and recurrent neural network for load-following operation of a PWR is presented by Boroushaki et al. ${ }^{28}$ This is an on-line intelligent controller that is capable of updating real plant data at any time interval for capturing any process dynamics not included in the training set of the neural network. The approach of Boroushaki et al. utilizes the DYNCO code designed to simulate the dynamic behavior of the Russian 3000-MWt PWR 
for testing and tuning of the controller. Most of the works on the load-following operations of nuclear reactors use a single-input single-output (SISO) model, whereas a multi-input multi-output (MIMO) model is used in this study in identification of nonlinear complex dynamics of the nuclear reactor. The performance of the controller has been evaluated through computer simulations.

\subsection{Emergency Operating Procedure}

The role of operators in the control room of nuclear reactors has become more and more important with the increasing size and complexity of NPPs. The operator's task is to maintain safety and reliability of an NPP. To ensure that this is achieved, it is necessary to increase the automation of operating procedures in NPPs, including those employed in abnormal and emergency situations. It is generally presumed that automation will enhance overall system reliability by reducing or removing the need for human action and that the operator will be freed from tasks that are routine, tedious, physically demanding, or difficult. The event-based approach developed in 1970s for operation and safety management of NPPs especially when operator intervention is warranted, proved to be efficient and easy to follow. However, the following weaknesses were identified in the TMI accident:

- Too much responsibility was placed on the operator to identify the abnormality quickly and properly and then to respond using an event-oriented procedure.

- Emphasis was placed on maintaining the plant availability while critical safety functions were ignored.

- Response procedures led to conflict with objectives when multiple events were identified.

- It was not possible to a priori define all unanticipated situations and abnormal events or combinations.

These weaknesses can be overcome by developing a symptom-oriented approach, which again suffers from inherent complexity. Another shortcoming of the symptom-oriented approach is that the maintenance of plant safety may lead to continued degradation of plant operation. Reported studies indicate that many expert systems have been developed to improve the complexities of the reactor operating procedures but that few have been tested in an NPP simulator or employed in practice.

In an effort to minimize the load on the operator during emergencies in an NPP, the automation of operating procedures using logic terminology and logic diagrams has been investigated by Husseiny et al. ${ }^{29}$ The prototype system has been tested for downpower and loss of forced circulation in a PWR. It is reported that such automation is feasible, provided that fault-tolerant software and hardware become available for design of controllers.

A small-scale prototype practical knowledge-based operator support system has been developed by Fujita et al. ${ }^{30}$ for a Japanese PWR. The system utilizes the plant abnormality model approach, as opposed to methods based on a normative model. The system consists of a decision support system and an intelligent interface. The decision support system consists 
of the following: a status evaluator, a diagnosis system, a decision maker, and a computational module. The functions of each of these modules can be explained as follows.

1. Status evaluator: The status evaluator monitors component status, detects and verifies anomalous symptoms, identifies possible failures, and generates qualitative data used by the system.

2. Diagnostic system: The diagnosis system identifies the causes of anomalous symptoms that may exist.

3. Decision maker: The function of the decision maker is to generate guidance that dynamically fits the plant status by selecting and synthesizing procedures for the plant status from among existing operational procedures.

4. Computational module: The computational module is an assembly of codes that calculates reference data and performs numerical calculations.

The intelligent interface adaptively makes access to the most useful information easier to obtain at any given moment. The system possess features that include monitoring of automatic component actions, early fault detection, diagnosis, operational guidance, and follow-up monitoring of operational actions recommended by the system. The system functionalities are verified for SGTR, where SGTR is simulated with a high-fidelity real-time plant simulator. The prototype system is upgraded to a full-fledged system for actual implementation.

An integrated decision support system has been developed by Kang et al. ${ }^{31}$ to provide the plant operators timely and proper guidelines under abnormal and emergency situations. This integrated operator decision aid support (IODAS) system performs four tasks: For example, signal validation and management, alarm processing, failure diagnostics, and dynamic emergency procedure tracking. This effort is undertaken to develop a system that will assist the operator in the decision-making process and ensure the safety of an NPP. The IODAS is based on the following three concepts:

1. When an NPP is in a normal operating condition, the system condition should be maintained to prevent an abnormal state.

2. If an NPP is in an abnormal state, alarm processing and failure diagnosis should be used to prevent a reactor trip.

3. If a reactor is tripped, early and safe plant shutdown should be achieved by employing emergency operating procedures.

The system is being implemented on a SUN-4/75 workstation using C and Quintus Prolog language, and its functionalities are verified with a full-scope real-time simulator. Test results show that IODAS is capable of diagnosing a plant failure quickly and of providing an operator guideline with fast response time. 
An intelligent control scheme is suggested by Choi, et $\mathrm{al}^{32}$ in an effort to automate the emergency procedure for optimal shutdown in a PWR. In this proposed method, fuzzy logic technique is used to adaptively adjust the controlled parameters for optimal cooldown and depressurization. The performance of the proposed control system is validated by integrating the control system with a microsimulator of Kori Unit 2. The tests are conducted with SGTR events of the Kori simulator. The results of the tests indicate that the automated emergency operation has successfully driven the plant at full power to a cold shutdown state with all operational constraints satisfied.

A decision-making technique based on fuzzy logic for the evaluation of accident management strategies in an NPP has been suggested by Jae and Moon. ${ }^{33}$ The approach in this study contains three major objectives:

1. representation of the decision problem,

2. fuzzy set evaluation of the decision alternatives, and

3. selection of the optimal alternatives.

Identification of decision goals and setting of the decision alternatives, identification of a set of decision criteria, and building a hierarchical structure of the decision problem are the activities that are performed in the representation of the decision problem section. The fuzzy set evaluation includes activities such as choosing the sets of the preference ratings, evaluating importance weights of the criteria, and aggregating the weights of decision criteria. The selection of optimal alternatives is accomplished through prioritizing of the decision alternatives using the aggregated assessments and choice of the decision alternatives with highest priority as the optimal. The proposed system is evaluated with successful results in the selection of the best accident management strategy for multiple decision alternatives in an NPP.

\subsection{Alarm Processing System}

In NPPs, an alarm system is provided to indicate abnormal situations and to aid in operator decision making. Due to the large size and the complexity of NPPs, enormous data influxes are present when a malfunction occurs. In such situations, the plant operators cannot diagnose the fault and process it quickly and properly. Earlier alarm systems, such as the annunciator system developed by Westinghouse Electric Corporation and the disturbance analysis system based on cause-consequence trees developed by Electric Power Institute, lack flexibility. In an effort to assist operators in NPPs in identifying primary causal alarms and in diagnosing the fault using modern technologies, several studies have been conducted $^{34-39}$ to automate alarm processing and diagnosis of NPPs.

Yang and Chang ${ }^{34}$ have suggested a prototype alarm processing system that is developed using model-based reasoning and object-oriented representation of the alarm with general 
cause-consequence check rules. This alarm processing system, which filters and suppresses unnecessary and potentially misleading alarms, is validated with an NPP simulator.

To assist the operator in identifying the primary causal alarm among multiple alarms and in diagnosing the plant malfunction promptly, Cheon et al. ${ }^{35}$ and Cheon et al. ${ }^{36}$ have investigated alarm processing systems using expert systems and neural networks, respectively. A prototype system of each has been developed, and encouraging results were observed when a number of case studies were performed on the Kori-2 NPP in South Korea.

A computerized annunciator system suggested by Naito and Ohtsuka ${ }^{37}$ is capable of extracting relevant alarms from the large number of alarms and of providing the operator with a clear overview of the process status on a CRT in a nuclear reactor control room. The alarm processing is realized using logical expressions for an alarm generation model generated by a human expert and is implemented with a process computer. The performances of the proposed system are validated on a full-scope BWR plant simulator with satisfactory results.

Progress toward further automation in alarm processing in NPPs is found in the published work of Choi et al. ${ }^{38}$ Choi et al. describe an on-line alarm processing system referred to as the alarm filtering and diagnostic system (AFDS). This system uses a fuzzy expert system that performs three tasks: For example, alarm filtering, system-wide alarm diagnosis, and alarm prognosis. The AFDS generates clear alarm pictures without any overlapping of alarms, provides system-wide failure information through alarm filtering and diagnosis, and enables operator-related operating procedures as well as diagnostic results. Another unique and important feature of this system is that it provides information about early failure detection or early plant abnormalities by providing trend information and predicted future values of some critical safety parameters in its prognosis stage. The Levinson algorithm is used to predict the future values of safety parameters. The AFDS has been proven successful after tests were conducted on the full-scope simulator of Yonggwang Units 1 and 2.

The study conducted by Park and Seong ${ }^{39}$ is focused on developing an integrated knowledgebase tool for knowledge acquisition and verification of an NPP dynamic alarm processing system. Document analysis method and extended colored Petri net matrix analysis with backward simulation are used to develop the knowledge acquisition and knowledge verification, respectively. The alarm knowledge was acquired more easily and consistently from knowledge of sensors when the tool was tested on the coolant system of Unit 3 of Kori NPP in Korea.

\subsection{Optimal Fuel Loading}

In-core fuel management continues to be among the main concerns of the NPP operators. The optimization of fuel loading patterns improves the cost-effectiveness of the overall plant operation and guarantees safety during the operation. An optimal loading pattern ensures that the local power peaking factor $\left(P_{\max }\right)$ is lower than a predetermined value during the "on" cycle and that the effective multiplication factor $\left(k_{e f f}\right)$ is maximized to extract the 
maximum energy. Over the last two decades, many researchers have attempted automatic optimization of the fuel reloading pattern by using various techniques such as direct search algorithms, simulated annealing methods, and linear programming. Because of the limitations of their search algorithms, none of these approaches ensure the global optimum solution. To resolve these limitations, researchers have explored artificial intelligence techniques such as ANNs, fuzzy-rule-based systems (FRBSs), genetic algorithms, and any combinations of these in fuel-reload-optimization activities. ${ }^{40-46}$

An optimal fuel loading pattern system has been proposed by Kim et al. ${ }^{40}$ that utilizes a backpropagation neural network to quickly predict core parameters, $P_{\max }$ and $k_{\text {eff, }}$, at the beginning-of-cycle for given fuel loading patterns. Researchers have claimed that this system predicts the core parameters several hundred times faster than the reference numerical code but that the prediction accuracy is not sufficient. This work has been further extended by Kim et al. ${ }^{41}$ in an attempt to improve the accuracy of the core parameter predictions. The fuel-shuffling system developed by Kim et al. ${ }^{41}$ was tested to generate the fuel loading pattern of cycle 1 of Kori-1 PWR. This technique takes advantage of the very fast prediction capability of an ANN and the very effective searching capability of an FRBS. The FRBS is used in order to achieve more effective and faster searching of loading patterns that satisfy $P_{\text {max }}$ requirements. The ANN, which utilizes a backpropagation network, predicts $P_{\max }$ and $k_{\text {eff }}$ for the "good" patterns generated by the FRBS. The fuzzy rule is optimized according to the prediction results by modification of its membership function. The system is developed using Quintus Prolog and C languages and installed on a SUN 4/75 workstation. It generates few patterns that are better than the reference pattern, and one can select from among them according to one's experience and knowledge. The system fails to generate the global optimal pattern but instead produces a local optimal pattern.

The optimum fuel loading pattern system called CIGARO, developed by using genetic algorithms, was demonstrated by DeChaine and Feltus. ${ }^{42}$ One salient feature of the CIGARO system is that it includes a code-independent interface that allows the use of any reactor physics code to evaluate the loading pattern. The interface program serves as translator between the genetic algorithm code and the reactor physics code. Basically, the interface program changes the output from one program into a format so that the other program can use it. The genetic algorithm portion of the code is programmed in $\mathrm{C}++$, and the interfaces are made with standard UNIX tools. The CIGARO system is tested with CASMO-3/SIMULATE-3 reactor physics code to optimize the loading pattern of a Westinghouse PWR. The system is designed to work for both the BWR and the PWR but has been tested only with the PWR. Better optimization results were later obtained by incorporating expert knowledge on fuel management optimization by DeChaine and Feltus ${ }^{43}$ with the original genetic algorithm system developed by DeChaine and Feltus. ${ }^{42}$

A method of Hopfield network integrated with the classical technique such as simulated annealing has been proposed by Sadighi et al. ${ }^{44}$ to find the best configuration of fuel assemblies in a PWR. This study shows that a Hopfield network is effective in finding a local minimum solution that can be extended to seek for a global minimum by integrating a simulated annealing method. Hopfield and simulated annealing routines are developed using FORTRAN. The code is tested to find the optimum loading pattern for the Bushehr NPP in 
Iran, which is verified against the pattern proposed by the designer of the plant, and the results show that the solution found is very close to optimum.

Unlike Kim's fuel-shuffling system, ${ }^{41}$ Erdogan and Geckinli ${ }^{45}$ developed a computer algorithm called XCore that combines the powerful learning and mimicking abilities of ANN with the genetic algorithm's stochastic optimization capability to optimize fuel loading pattern. In this algorithm, the ANN predicts the power distribution in the core and the effective multiplication factor $\left(k_{\text {eff }}\right)$, which are used by the genetic algorithm to search for an optimum loading pattern to ensure safe and efficient reactor operation. The primary goal of this study is to automate the fuel loading pattern generation that satisfies reactor safety requirements and to minimize the operating costs by reducing the initial amount of fuel charged to the core and by increasing burnup. XCore is written in $\mathrm{C}++$ and runs under the LINUX operating system. The code has been tested successfully by using the data from the PWR Almaraz Nuclear Power Station in Spain, and the test results conform very well to those loading patterns suggested for the nuclear reactor core. The authors suggest that the capabilities of XCore can be enhanced further by including burnup and leakage-flux effects into the both ANN and genetic algorithm modules of the code.

An algorithm based solely on ANN is proposed by Faria and Pereira ${ }^{46}$ for the optimum loading pattern for a PWR. In this algorithm, the loading pattern is generated by ANN whereas the core parameters are calculated with the WIMS-D4 and CITATION-LD12 codes to satisfy the $P_{\max }$ constraint only. The developed procedure is able to generate a better loading pattern than the reference loading pattern but does not always guarantee a global optimum loading pattern.

\subsection{Fault Detection and Identification}

Fault detection and identification have always been of paramount importance to improve safety in nuclear reactor operations. Quick detection and accurate diagnosis of faults are extremely important to NPP safety because relatively simple misdiagnosis followed by inappropriate corrective action may lead to a more dangerous situation. Many studies have been reported in the literature investigating the ways to develop fault detection and diagnostic systems to address the above issues.

A fault diagnostic system for the emergency feedwater system (EFWS) of the Seabrook Nuclear Station has been developed by Obreja ${ }^{47}$ using expert systems. The proposed diagnostic system consists of a fault detection module and a fault diagnosis module. The symptom of malfunction is detected by the fault detection module by observing the qualitative variation of the output parameter, which is then propagated through the system model where all possible faults are generated. The knowledge base contains the qualitative information of the EFWS, which is used by the diagnostic process. The system has been tested only on a portion of the whole EFWS model.

One of the major drawbacks of using expert systems is that the monitored input variables can be degraded by noise, which can cause expert systems to deviate from the formulated 
conditions at critical decision levels. These systems may also require a significant amount of computation time and resources to progress through the many decision levels required to diagnose a fault. To alleviate this problem, a system using a self-optimizing stochastic learning algorithm ANN for fault detection and diagnosis has been suggested by Bartlett and Uhrig. ${ }^{48}$ In this study, the ANN is trained on normal operating conditions as well as on potentially unsafe conditions of an NPP. ANN is chosen by Bartlett because it has demonstrated improved diagnostic capabilities in situations in which data are noisy and incomplete. NPP simulation data from the Watts Bar Nuclear Power Station are used to test the algorithm. The ANN is taught seven accident scenarios of the NPP, such as total loss of off-site power, main feedwater line break, main steam line break, control rod ejection, hotleg loss of coolant, cold-leg loss of coolant, and steam generator tube leak. The data set for each scenario contains 27 plant process variables at 0.5 -s intervals for at least $250 \mathrm{~s}$. The results of the tests are depicted in graphical form for each of the above fault conditions for situations without noise present in data and for those with noise. The results demonstrated that the network responds very quickly to the onset of most of the accidents except in the case of steam generator leak; however, the network classifies this accident well in advance of the reactor scram.

On the other hand, a system that combines a neural network and knowledge processing has been proposed by Ohga and Seki ${ }^{49}$ to identify abnormal events that cause a nuclear reactor scram. The change pattern of plant variables in analog form is recognized by the neural network, which is then outputted as the candidate abnormal event. The knowledge processing reads the event code from the neural network and confirms the network result using a knowledge base for the plant data. It has been shown that the neural network can identify effectively any event on which it is trained, even in presence of white noise, and that it does not mistakenly identify any nontrained event. This method provides rapid identification using a small amount of knowledge. The system performance is validated through tests on the simulated results of a transient analysis program for a BWR. The data for neural network input includes reactor pressure, reactor water level, neutron flux, main steam flow rate, and feedwater flow rate. Ten events are selected along with a white noise, with a standard deviation of $10 \%$ for the test. The test results show that the neural network can identify a trained event when the plant condition differs from the trained one.

The work of Bartlett and Uhrig ${ }^{48}$ has been extended by Kim and Bartlett ${ }^{50}$ to address the issue of accuracy of the previous fault diagnosis algorithms. A method presented in ref. 50 provides an error bound and hence a figure of merit for the diagnostic system of NPPs developed previously by using ANN. For the following reasons, this error-bound prediction method utilizes a modified stacked generalization scheme based on cross-validation and nonparametric statistics. Cross-validation is very effective in selecting the best model for data (such as Fault diagnosis data) that are not normally distributed. Statistical modeling by nonparametric methods is more accurate for the type of data that the fault diagnosis system produces. The developed system includes an ANN advisor that detects and diagnoses faults and a stacked generalization technique to estimate the error bounds of the main ANN advisor. The data used for training the ANN are taken from the San Onofre Nuclear Power Plant (a 500-MW PWR), and the system is tested for 10 simulated transient situations containing 33 plant variables and 10 simulated normal operating conditions of the NPP. The 
root-mean-square error of each of the above cases is displayed in graphical form, demonstrating the effectiveness of the method and providing estimated error bounds for each of its diagnoses.

The error predictor network used in the above approach requires an additional input vector that doubles the total input space as compared with that of the ANN advisor. This increase in input space causes increase in complexity and additional difficulties in network training. Another disadvantage of this technique is that the error predictor network is fed by an input vector that is unrelated to the performance of the main ANN advisor. To overcome these drawbacks of the previous error bound estimator of an ANN advisor, Kim and Bartlett ${ }^{51}$ presented a scheme called "error estimation by series association," by replacing the previous error predictor network with a new network developed via a different technique. The error estimation in this approach is performed by a secondary neural network that is fed both the inputs for and the outputs of the ANN advisor. Encouraging results have been reported from the test on 33 transient scenarios of various severity levels and degraded noise conditions of the Duane Arnold Energy Center NPP ranging from a main steam line break to anticipated transient without scram. The developed ANN fault diagnosis system demonstrates increasing robustness to noise compared with other systems.

\subsection{Sensor Validation and Detection}

The sensor validation plays an important role in real-time NPP operations. Faulty and conflicting sensor readings may often degrade the performance of the control system, confuse operators, and lead to actions that may compromise the safety of an NPP.

Automation of the sensor validation process will be an important advancement for increased safety and higher autonomy in control functions of an NPP. The proposed method presented by Eryurek and Upadhyaya ${ }^{52}$ uses an adaptive backpropagation network for signal validation of an NPP. During the training period for the network, both steady-state and transient behavior can be included and new data can be incorporated by updating the network connection weight. The most important feature of the algorithm is the ability to adjust the sigmoidal threshold function and weight-updating terms progressively during the learning phase. Test results have indicated that the speed of convergence and accuracy of results can be improved by incorporating the above capability in the network. The algorithm is tested with the operational data from a four-loop Westinghouse PWR at startup for a period of approximately $36 \mathrm{~h}$. The predicted values can be used for sensor validation, process monitoring, and diagnostic applications. This work has been extended by Upadhyaya and Eryurek $^{53}$ to include additional features, which are listed below. The sigmoidal function can be changed on-line, all input and output signals can be scaled automatically, and a single hidden layer is sufficient (instead of the multiple hidden layers normally used). Startup data from EBR-II are used to illustrate the effectiveness of the approach. It is reported that the reactor power ( 0 to $100 \%$ ), the control rod position, the core exit temperature, and the secondary sodium outlet temperature of the intermediate heat exchanger are predicted with a higher degree of accuracy. 
A different approach that utilizes computer-aided techniques to automate the sensor validation process is suggested by Ning and $\mathrm{Chou}^{54}$ for sensor validation of NPPs. Ning and Chou have proposed a fault detection network that consists of sensors, mathematical models for sensor relations, and a decision/estimator unit — all linked in a fault-tree structure. The function of the decision/estimator is to discriminate minority sensors and isolate their readings. The data structuring is done using an entity relationship modeling technique, and data manipulation is accomplished via object-oriented programming. This scheme provides a tool to build the fault detection network in a systematic manner. The system is implemented in a personal computer, where the codes are written in $\mathrm{C}$. The tests have been conducted to evaluate the system with Taipower Company's Maanshan three-loop PWR in Taiwan, with encouraging results.

The process empirical modeling (PEM) technique is proposed by Holbert and Upadhyaya ${ }^{55}$ for the purpose of signal validation. The empirical models are developed by using data from different steady-state operations. The nonlinear multiple-output single-input model developed by this technique compares the sensor output with the measured signal to identify any anomaly. The main advantage of this method as compared with analytical methods lies in the reduction of effort required to create the models. The technique possesses the ability to replace a faulty measurement as an input to a control system. Holbert and Upadhyaya report that the PEM technique has the ability to automatically discard the signal that does not change with the output. This feature has proved to be a drawback in cases where a process variable is held constant throughout the operation of the plant, such as the flow rate of a reactor coolant system. The authors warn that this aspect must be accounted for in any practical application. The technique employs fuzzy S-curve to identify signal anomaly by comparing the deviation of the measured and predicted signals with the signal tolerance. If the measured value is found to be inaccurate, the predicted value is used for input to the control system or as a display for the operator in real time. The method is applied to operational data from a four-loop Westinghouse PWR and the EBR-II. A total of 9 variables of the PWR and 19 variables of the EBR-II are modeled. The test results indicate that errors of the prediction accuracy of the PEM are 0.09 to $3.9 \%$ and 0.06 to $0.6 \%$ for the PWR and EBR-II variables, respectively. The PEM has proved to be highly useful as an independent estimate of process conditions.

For signal validation, Fantoni and Mazzola ${ }^{56}$ suggest a pattern recognition-ANN-based system that is capable of identifying abnormal condition. The major accomplishment of this work is that the system can validate process signals in case of multiple-failure scenarios, both in steady-state and transient conditions. The signal validation process is accomplished using multilayered supervised ANN with a backpropagation algorithm. The training data for the ANN is obtained from a BWR simulator (APROS) that contains both transient and steadystate conditions with added noise ( $2 \%$ superimposed standard deviation). The test results, including the case of double fault, indicate that the system estimates the process signals satisfactorily with a maximum error of $5 \%$ (below $3 \%$ in most of the cases).

A sensor validation system proposed by Heger et al. ${ }^{57}$ is based on a fuzzy logic approach and includes the functions such as instantaneous signal validation and measurement of long-term sensor status when two or three redundant sensors are used. The first function provides 
information about the current status of the sensors, which is then used to compute the best estimate of the measured variable. The second function uses a frequency fuzzy variable to determine the signal condition over a specific period. The instantaneous signal validation is important in detection of any catastrophic fault in a sensor, whereas the long-term evaluation will discern a slowly developing fault such as drift. The generalized likelihood ratio (GLR) and sequential probability ratio test (SPRT) methods are used for the instantaneous and longterm signal validation, respectively. The GLR technique is very useful in detecting abrupt changes or failures of the system and provides a single best estimate of a particular variable by comparing the reading with the sensor readings of the variable from other counterparts. The SPRT provides a systematic procedure for fault detection and isolation. The decision is based on the information gathered from the past measurement history and the current observation. The system is tested by analyzing synthetically generated data, which are produced to mimic various possible scenarios that may occur during a sensor malfunction or normal plant operation. A Gaussian noise of mean value zero and standard deviation of unity is superimposed on the signals. It is shown that the technique is effective in evaluating the sensor status for both instantaneous and long-term signal validation.

Earlier reported methods in signal validation using neural networks may require a large number of input signals to estimate the output signal. The selection of input signals plays a vital role in the performance of such networks. The training time for neural networks increases exponentially as the number of input signals becomes larger. A reliable and effective sensor detector proposed by $\mathrm{Na}$ et al. ${ }^{58}$ automatically optimizes the number of required input signals and hence reduces the neural network training time. The technique utilizes a fuzzy neural network with an optimal structure constructor. Correlation analysis and genetic algorithms are also combined in this technique to perform the optimal selection of proper input signals, whereas the fuzzy neural network estimates the output signals from the optimum input signals. The genetic algorithm is also used here to automatically generate the optimum number of fuzzy rules. The residual error is generated by taking the difference between the estimated and the measured signal, and, subsequently, the degree of degradation and continuous behavior of the sensor may be determined with SPRT applied to the residual error. The proposed technique is applied to monitor the steam-generator water level, hot-leg temperature, and the ex-core neutron flux sensors in Yonggwang Unit 3 and 4 PWRs. The test results show that the performance of this algorithm is superior to other existing algorithms.

\section{CONCLUSIONS}

With ever-increasing demands for quality improvement, reliability, safety, and cost reduction placed on NPPs there is a trend toward autonomous operations in which the tasks of performance monitoring, early fault detection and diagnosis, automated alarm processing, emergency operating procedures, etc., can be integrated. Autonomous control systems must perform well under significant uncertainties in the plant and the environment for extended periods of time, and they must be able to compensate for system failures without external intervention. In this study, we have tried to collect information on ongoing efforts, current 
status, and future trends to achieve higher automation of NPP operations. Nuclear engineers are constantly engaged in developing controllers for NPPs with higher autonomous behavior. Clearly, recent developments show that intelligent techniques such as fuzzy logic, neural networks, genetic algorithms, expert systems, and others provide additional tools that significantly enlarge the range of problems that can be solved. An increasing number of studies have been conducted in recent years that contain intelligent agents such as fuzzy logic, neural networks, genetic algorithms, etc., to achieve higher autonomy in complex plant operations like NPP, and it has been proven that such systems have the potential to operate with high levels of performance and reliability with minimum operator intervention. There is a broad consensus that a reasonable degree of autonomy is more easily achievable by incorporating intelligence components in the conventional control systems. Nuclear engineers also share the opinion that more studies must be focused on the stability analysis of intelligent control systems. 


\section{REFERENCES}

1. Uhrig, R. E., "Opportunities for Automation and Control of the Next Generation of Nuclear Power Plants," Nuclear Technology, 88, 157-165 (Nov. 1989).

2. Antsaklis, P. J., Passino, K. M., and Wang, S. J., "An Introduction to Autonomous Control," IEEE Control System Magazine, 5-13 (June 1991).

3. Antsaklis, P. J., and Passino, K. M., An Introduction to Intelligent and Autonomous Control, Kluwer Academic Publishers, Boston, 1992.

4. Antsaklis, P. J., "Intelligent Learning Control," IEEE Control System Magazine, 5-7 (June 1995).

5. Narendra, K. S., "Intelligent Control," IEEE Control System Magazine, 39-40 (June 1991).

6. Astrom, K. J., "Where Is the Intelligence in Intelligent Control?" IEEE Control System Magazine, 37-39 (June 1991).

7. Chaudhuri, T. R., Hamey, L. G. C., and Bell, R. D, "From Conventional to Autonomous Intelligent Methods," IEEE Control System Magazine, 78-84 (Oct. 1996).

8. Passino, K. M., "Intelligent Control for Autonomous Systems," IEEE Spectrum, 55-62 (June 1995).

9. Passino, K. M., and Ozguner, U., "Intelligent Control: From Theory to Application," IEEE Expert, 11(2), 28-30 (April 1996).

10. Passino, K. M., "Bridging the Gap between Conventional and Intelligent Control," IEEE Control System Magazine, 12-18 (June 1993).

11. Astrom, K. J., "Toward Intelligent Control," IEEE Control System Magazine, 60-64 (April 1989).

12. Shoureshi, R., "Learning and Decision Making Intelligent Control Systems," IEEE Control System Magazine, 34-37 (Jan. 1991).

13. Antsaklis, P. J., and Kantor, J. C., "Intelligent Control for High Autonomy Process Control Systems," pp. 37-46 in First International Conference on Intelligent Systems in Process Engineering: Proceedings of the Conference Held at Snowmass, Colorado, July 9-14, 1995. American Institute of Chemical Engineers, New York, 1996.

14. Williams, J. G., and Jouse, W. C., "Intelligent Control in Safety Systems: Criteria for Acceptance in the Nuclear Power Industry," IEEE Transactions on Nuclear Science, 40(6), 2040-2044 (Dec. 1993).

15. Ruan, D., "Intelligent Systems in Nuclear Applications," International Journal of Intelligent Systems," 13, 115-125 (1998).

16. Cha, K., "A Real-Time Knowledge-Based Simulation for Process Control and Monitoring of a Nuclear Power Plant," pp. 347-350 in First International Conference on Intelligent Systems in Process Engineering: Proceedings of the Conference Held at Snowmass, Colorado, July 9-14, 1995. American Institute of Chemical Engineers, New York, 1996.

17. Doraiswami, R., Stevenson, M., and Diduch, C. P., "Autonomous Control Systems: Monitoring, Diagnosis and Tuning," IEEE Transactions on Systems, Man, and Cybernetics: Part A, 26(5), 646-655 (Sept. 1996). 
18. Ben-Abdennour, B., and Lee, K. W., "An Autonomous Control System for BoilerTurbine Units," IEEE Transactions on Energy Conversion, 11(2), 401-406 (June 1996).

19. March-Leuba, J., and Wood, R. T., "Development of an Automated Approach to Control System Design," Nuclear Technology, 141, 45-53 (Jan. 2003).

20. Berkan, R. C., Upadhyaya, B. R., and Tsoukalas, L. H., "Advanced Automation Concepts for Large-Scale Systems," IEEE Control Systems Magazine (Oct. 1991).

21. Sekimizu, K., Araki, T., and Kawakami, S., "Knowledge Representation for Automated Boiling Water Reactor Startup," Nuclear Technology, 100, 295-309 (Dec. 1992).

22. Ramaswamy P., Edwards, R. M., and Lee, K. Y., "An Automatic Tuning Method of a Fuzzy Logic Controller for Nuclear Reactors," IEEE Transactions on Nuclear Science, 40(4), 1253-1262 (Aug. 1993).

23. Ku, C. C., Lee, K. Y., and Edwards, R. M., "Improved Nuclear Reactor Temperature Control Using Diagonal Recurrent Neural Networks," IEEE Transactions on Nuclear Science, 39(6), 2298-2304 (Dec. 1992).

24. Na, M. G., and Upadhyaya, B. R., "A Neuro-Fuzzy Controller for Axial Power Distribution in Nuclear Reactors," IEEE Transactions on Nuclear Science, 45(1), 5967 (Feb. 1998).

25. Stengel, R. F., "Intelligent Failure-Tolerant Control," IEEE Control Systems Magazine, 14-23 (June 1991).

26. Rauch, H. E., "Intelligent Fault Diagnosis and Control Reconfiguration," IEEE Control Systems Magazine, 6-12 (June 1994).

27. Khajavi, M. N., Menhaj, M. B., and Suratgar, A. A., "A Neural Network Controller for Load Following Operation of Nuclear Reactors," Annals of Nuclear Energy, 29, 751760 (2002).

28. Boroushaki, M., Ghofrani, M. B., Lucas, C., and Yazdanpanah, M. J., "An Intelligent Nuclear Reactor Core Controller for Load Following Operations, Using Recurrent Neural Networks and Fuzzy Systems," Annals of Nuclear Energy, 30, 63-80 (2003).

29. Husseiny, A. A., Sabri, Z. A., Packer, D., Holmes, J. W., Adams, S. K., and Rodriguez, R. J., "Operating Procedure Automation to Enhance Safety of Nuclear Power Plants," Nuclear Science and Design, 110, 277-297 (1989).

30. Fujita, Y., Tohyama, M., Yanagisawa, I., Ida, T., and Arikawa, H., "Designing a Knowledge-Based Operator Support System for Practical Applications," Nuclear Technology, 95, 116-128 (July 1995).

31. Kang, S. K., Kim, H. G., Chang, S. H., Jeong, H. K., and Park, S. D., "Development Strategies on an Integrated Operator Decision Aid Support System for Nuclear Power Plants," IEEE Transactions on Nuclear Science, 40(4), 1295-1304 (Aug. 1993).

32. Choi, S. S., Chang, S. H., and Lee, D. H., "Automating Strategies of Emergency Operation for Optimal Shutdown in Pressurized Water Reactors," IEEE Transactions on Nuclear Science, 45(1), 17-29 (Feb. 1998).

33. Jae, M., and Moon, J. H., "Use of Fuzzy Decision-Making Method in Evaluating Severe Accident Management Strategies," Annals of Nuclear Energy, 29, 1597-1606 (2002).

34. Yang, J. O., and Chang, S. H., "An Alarm Processing System for a Nuclear Power Plant Using Artificial Intelligence Techniques," Nuclear Technology, 95, 266-271 (Sept. 1991). 
35. Cheon, S. W., Chang, S. H., and Chung, H. Y., "Development Strategies of an Expert System for Multiple Alarm Processing and Diagnosis in Nuclear Power Plants," IEEE Transactions on Nuclear Science, 40(1), 21-30 (Feb. 1993).

36. Cheon, S. W., Chang, S. H., and Chung, H. Y., "Application of Neural Networks to Multiple Alarm Processing and Diagnosis in Nuclear Power Plants," IEEE Transactions on Nuclear Science, 40(1), 11-20 (Feb. 1993).

37. Naito, N., and Ohtsuka, S., "Intelligent Alarm-Processing System for Nuclear Power Plants," Nuclear Technology, 109, 255-264 (Feb. 1995).

38. Choi, S. S., Kang, K. S., Kim, H. G., and Chang, S. H., "Development of an On-Line Fuzzy Expert System for Integrated Alarm Processing in Nuclear Power Plants," IEEE Transactions on Nuclear Science, 42(4), 1406-1418 (Aug. 1995).

39. Park, J. H., and Seong, P. H., "An Integrated Knowledge Base Development Tool for Knowledge Acquisition and Verification for NPP Dynamic Alarm Processing Systems," Annals of Nuclear Energy 29, 447-463 (2002).

40. Kim, H. G., Chang, S. H., and Lee, B. H., "Pressurized Water Reactor Core Parameter Prediction Using an Artificial Neural Network," Nuclear Science and Engineering, 113, 70-76 (1993).

41. Kim, H. G., Chang, S. H., and Lee, B. H., "Optimal Fuel Loading Pattern Design Using an Artificial Neural Network and a Fuzzy Rule-Based System," Nuclear Science and Engineering, 115, 152-163 (1993).

42. DeChaine, M. D., and Feltus, M. A., "Nuclear Fuel Management Optimization Using Genetic Algorithms," Nuclear Technology, 111, 109-114 (July 1995).

43. DeChaine, M. D., and Feltus, M. A., "Fuel Management Optimization Using Genetic Algorithms and Expert Knowledge," Nuclear Science and Engineering, 124, 188-196 (1996).

44. Sadighi, M., Setayeshi, S., and Salehi, A. A., "PWR Fuel Management Optimization Using Neural Networks," Annals of Nuclear Energy, 29, 41-51 (2002).

45. Erdogan, A., and Geckinli, M. "A PWR Reload Optimization Code (XCore) Using Artificial Neural Networks and Genetic Algorithms," Annals of Nuclear Energy, 30, 35-53 (2003).

46. Faria, F. E., and Pereira, C., "Nuclear Fuel Loading Pattern Optimisation Using a Neural Network," Annals of Nuclear Energy, 30, 603-613 (2003).

47. Obreja, I., "Qualitative Knowledge in a Diagnostic Expert System for Nuclear Power Plant Safety," pp. 131-134I in Proceedings of IFAC SAFECOMP'90, Pergamon Press, Oxford, United Kingdom, 1990.

48. Bartlett, E. B., and Uhrig, R. E., "Nuclear Power Plant Status Diagnostics Using an Artificial Neural Network," Nuclear Technology, 97, 272-281 (March 1992).

49. Ohga, Y., and Seki, H., "Abnormal Event Identification in Nuclear Power Plants Using a Neural Network and Knowledge Processing," Nuclear Technology, 101, 159-163 (Feb. 1993).

50. Kim, K., and Bartlett, E. B., "Error Prediction for a Nuclear Power Plant FaultDiagnostic Advisor Using Neural Networks," Nuclear Technology, 108, 283-297 (Nov. 1994).

51. Kim, K., and Bartlett, E. B., "Nuclear Power Plant Fault Diagnosis Using Neural Networks with Error Estimation by Series Association," IEEE Transactions on Nuclear Science, 43(4), 2373-2388 (Aug. 1996). 
52. Eryurek, E., and Upadhyaya, B. R., "Sensor Validation for Power Plants using Adaptive Backpropagation Neural Network," IEEE Transactions on Nuclear. Science, 37(2), 1040-1047 (April 1990).

53. Upadhyaya, B. R., and Eryurek, E., "Application of Neural Networks for Sensor Validation and Plant Monitoring," Nuclear Technology, 97, 170-176 (Feb. 1992).

54. Ning, J. N., and Chou, H. P., "Construction and Evaluation of Fault Detection Network for Signal Validation," IEEE Transaction on Nuclear Science, 39(4), 943-947 (1992).

55. Holbert, K. E., and Upadhyaya, B. R., "Empirical Process Modeling Techniques for Signal Validation," Annals of Nuclear Energy, 21(7), 387-403 (1994).

56. Fantoni, P. F., and Mazzola, A., "A Pattern Recognition-Artificial Neural Networks Based Model for Signal Validation in Nuclear Power Plants," Annals of Nuclear Energy, 23(13), 1069-1076 (1996).

57. Heger, A. S., Holbert, K. H., and Ishaque, A. M., "Fuzzy Associative Memories for Instrument Fault Detection," Annals of Nuclear Energy, 23(9) 739-756 (1996).

58. Na, G. M., Sim, Y. R., Park, K. H., Lee, S. M., Jung, D. W., Shin, S. H., Upadhyaya, B. R., Zhao, K., and Lu, B., "Sensor Monitoring Using Fuzzy Neural Network with an Automatic Structure Constructor," IEEE Transactions on Nuclear Science, 50(2), 241250 (April 2003). 